\title{
RECTIFICATION TECHNOLOGY FOR PREPARING ESPECIALLY PURE CRYOGENIC PRODUCTS
}

\author{
E. I. Borzenko, L. A. Akulov, \\ and A. V. Zaitsev
}

Highly effective technology has been developed providing preparation of various especially pure cryogenic products, production schemes are provided for automated rectification modules, and a procedure has been developed for the design.

Currently, the main cryoproducts (oxygen, nitrogen, and argon), prepared with air separation, are used extensively in various branches of science and technology. In a number of production processes, particularly high specifications are laid down for purity of these products, in accordance with which the content of individual impurities should be several orders of magnitude lower than those that are permitted in relation to these products in existing GOST and TU.

These high specifications are made for purity of a number of gases, for example hydrogen, helium, neon, and carbon dioxide. The limiting overall content of microimpurities for individual components in a number of cases should not exceed $10^{-5}-10^{-7} \%$ (vol.).

In low-temperature technology in order to prepare particularly pure gases methods of low-temperature rectification and adsorption are used. Depending on the composition of the mixture being separated, its parameters and volume in a NTU, designated for preparing especially pure gas, it is possible to use one of these methods or two successively.

In order to prepare technically pure air separation products (such nitrogen, oxygen and argon), cryogenic air separation units (ASU) are used, in whose separation assembly a method of low-temperature rectification is used. However, in those cases when it would be necessary to separate one of the components in especially pure form, use of standard ASU appears to be undesirable. This is explained by the fact that in a production scheme the device is quite complicated and its operation presents particular difficulties.

In these cases, the most rational solution of this problem may be achieved as a result of preparing particularly pure cryoproducts from those of technical purity in special automated cryogenic modules. This makes it possible to organize production of especially pure cryoproducts not only for those products where technically pure products of air separation are obtained, but also directly for the user of particularly pure cryoproducts.

In the Cryogenic Technology Department of the St. Petersburg State University of Low Temperatures and Food Technology highly efficient technology has been developed making it possible to provide preparation of various especially pure cryoproducts. As a result of this scientific research with the aim of cryoproduct preparation in liquid and gaseous states with the minimum amount of impurities production schemes have been proposed for automatic rectification modules and a procedure for designing them has been developed.

During analysis of various design solutions, the main energy properties of the purification process have been determined making it possible to evaluate the efficiency of the design solutions adopted.

The technology developed for preparing especially pure cryoproducts has been put into industrial production.

St. Petersburg State University of Low Temperatures and Food Technology, St. Petersburg, Russia. Translated from Khimicheskoe i Neftegazovoe Mashinostroenie, No. 10, p. 22, October, 2009. 